\title{
A Study on the Adverse Drug Reactions Induced by Anti Epileptic Drugs in the Epileptic Patients
}

\author{
Keerthy Jayalekshmi, Kavitha Palanisamy*, Sambathkumar Ramanathan, Sruthi Akela \\ Department of Pharmacy Practice, J.K.K. Nattraja College of Pharmacy, Kumarapalayam, Erode, Affiliated to Dr. M.G.R. Medical University, Tamilnadu- \\ 638183, India.
}

\section{ARTICLE INFO}

\section{Article history:}

Received on: 08/01/2016

Revised on: 16/02/2016

Accepted on: 11/03/2016

Available online: 28/05/2016

Key words:

Epilepsy, anti epileptic drug, adverse drug reactions.

\begin{abstract}
Epilepsy is a chronic disorder of the brain that affects people worldwide. The aim of this work was to study the adverse drug reactions (ADRs) induced by the antiepileptic drugs (AEDs) in epileptic patients. This prospective study was carried out for six months, from April 2014 to September 2014, in a tertiary teaching care hospital, Erode. The study included epileptic patients, both male and female of all age groups, undergoing treatment with AEDs. A total of 180 epileptic patients were monitored, $60 \%$ were male and $40 \%$ were females. Majority of the study population were adults. Majority of the epileptic patients were affected with generalized seizure (46.66\%). Monotherapy was preferred more $(71.1 \%)$ over combination therapy $(34.44 \%)$. Sodium valproate $(24.4 \%)$ was the most prescribed drug in monotherapy followed closely by Phenytoin (18.3\%). Most prescribed two drug combination was of Sodium valproate and Carbamazepine (8.3\%) followed by Phenytoin and Phenobarbitone (6.67\%). Most common ADR was memory loss, hepatotoxicity, aggression and reduced learning. Sodium valproate was found to induce more number of ADRs followed by Carbamazepine and Phenytoin. Treatment with antiepileptic drugs was continued in all cases, except in three cases of skin rashes.
\end{abstract}

\section{INTRODUCTION}

Epilepsy is a relatively common condition characterized by a tendency for recurrent seizures, which is due to the disturbance of spread of electrical discharge of the cortical neurons. Up to $80 \%$ of people with epilepsy are able to control their condition with anti epileptic drugs (AEDs). The type of drug therapy prescribed depends on the type of seizure, the underlying cause of the epilepsy, age of the patient and possible side effects. Treatment usually starts with one drug at a low dose. The dose is then increased slowly. In most patients, epilepsy remits over a period of years and drug therapy may be withdrawn slowly (Abbashar, 2010). According to World Heath Organization (WHO) statistics, approximately 50 million people

\footnotetext{
* Corresponding Author

Kavitha Palanisamy, M.Pharm Lecturer, J.K.K.Nattraja College of pharmacy, Kumarapalayam, Erode, Tamil Nadu, India. Ph.No: +91-9042252212; Email: kavitha.p119@gmail.com
}

worldwide have epilepsy (Soha et al., 2011). In India, the prevalence rate of epilepsy ranges between 4.15 and 7.03 per 1000 population. In newly diagnosed cases, $60 \%$ are partial and $40 \%$ generalized. Epilepsy adversely affect the psycho-social status and quality of life of patients. General approaches for epilepsy management involves identification of goals and development of care plan.

The ultimate goal is seizures freedom without adverse effects of medication and improved quality of life (Juny et al., 2013). Epilepsy often requires long-term AED therapy. However, prolonged AED administration is associated with a number of problems such as behavioral and psychiatric disorders, metabolic and endocrine disorders, idiosyncratic reactions, and drug interaction effects. Although some studies suggest that patients with epilepsy treated with AEDs have an increased risk of fractures, low bone mineral density, and abnormalities in bone metabolism, skeletal diseases associated with long-term AED treatment are seriously unrecognized (Lee et al., 2013). 
Epilepsy is a long-term disease which requires chronic therapy. Due to the long duration of treatment, various adverse reactions (ADRs) are seen, which require change of medication and monitoring (Amrita et al., 2012). This study focuses upon on the chance of ADR development in the epileptic patients. The aim was to identify, record and assess the adverse drug reactions induced by the antiepileptic drugs in epileptic patients.

\section{MATERIALS AND METHOD}

This was a prospective study, carried out for a period of six months, from April 2014 to September 2014, in a tertiary teaching care hospital, Erode. The study received clearance from Institutional ethical committee. Data of epileptic patients of all age group, both sexes, including in-patients and out-patients were collected after explaining the purpose of the study to the subjects verbally.

Epileptic patients taking drugs other than AEDs, epileptic patients with skin diseases, pregnant epileptic patients and psychiatric patients were excluded. The details of the epilepsy cases, including laboratory blood analysis, liver function tests and electrolyte profile values were collected in data entry forms. Patients were interviewed to assess cognitive impairment. The identified ADRs were entered in the ADR form and the causality assessment of ADRs was done using Naranjo's scale.

\section{STATISTICAL ANALYSIS}

The subjects were classified into patients presenting and not presenting ADRs. The categorical variables were reported in percentage and continuous variables were expressed as mean \pm SD. The relationship between age and the number of detected ADRs was analyzed using chi square test. To examine for any possible association between the number of ADRs with gender, and with AED therapy (monotherapy, combination therapy), independent $t$ test was used. One-way analysis of variance was applied to assess the association between type of seizure and the number of ADRs. $\mathrm{P}<0.05$ was considered to be statistically significant. For statistical analysis, the graph pad prism version 6 was used.

\section{RESULT AND DISCUSSION}

A total of 180 epileptic patients were included. Table 1 describes the demographic details and clinical profiles of the study subjects. Out of the 180 patients, $60 \%$ were males and $40 \%$ were females. The lesser number of females may be due to unequal sex ratio higher illiteracy, poorer understanding of the disease and treatment, social stigma, and the need for male relative to consent and accompany the females for hospital visit (Juny et al., 2013; Manzur et al., 2013). Patients from all the age groups were included in the study. Patients between age group 0-14 years were dominant with $59(32.78 \%)$ subjects followed by patients from the age group $15-29$ years $(25.55 \%)$. $62.22 \%$ subjects were outpatients and $37.78 \%$ were in-patients. Adult patients dominated overall and this coincides with other studies also (Juny et al., 2013).

Table 1: Demographic profile and clinical details of the study subjects $(\mathrm{n}=180)$.

\begin{tabular}{clc}
\hline SI.No & \multicolumn{1}{c}{ Parameter } & No. of patients \\
\hline 1. & Gender & $108(60 \%)$ \\
& Male & $72(40 \%)$ \\
\hline 2. & Female & \\
& Age (years) & $59(32.78 \%)$ \\
& $0-14$ & $46(25.55 \%)$ \\
& $15-29$ & $31(17.22 \%)$ \\
& $30-44$ & $23(12.78 \%)$ \\
& $45-59$ & $21(11.66 \%)$ \\
\hline 3. & Ty-74 & \\
& GTPe of seizure & $84(46.66 \%)$ \\
& CPS & $35(19.44 \%)$ \\
& Myoclonic seizure & $28(15.55 \%)$ \\
& Unclassified seizure & $17(9.44 \%)$ \\
& Atonic seizures & $11(6.11 \%)$ \\
& Status epilepticus & $5(2.78 \%)$ \\
\hline 4. & Type of patient & $112(62.22 \%)$ \\
& Out patient & $68(37.78 \%)$ \\
\hline & In patient &
\end{tabular}

GTCS-Generalized tonic-clonic seizure, CPS-Complex partial seizure.

Majority of the study subjects were affected with generalized seizures and $84(46.66 \%)$ subjects had generalized tonic clonic seizure followed by $35(19.44 \%)$ cases of complex partial seizures and $28(15.55 \%)$ cases of myoclonic seizures. Other studies also describes generalized tonic clonic seizures to be the most common type of seizures among epileptic patients (Shobana et al., 2010; Juny et al., 2013; Manzur et al., 2013; Swetha et al., 2013).

Table 1: AED regimen used in subjects $(\mathrm{n}=180)$.

\begin{tabular}{lc}
\hline \multicolumn{1}{c}{ AED regimen } & No. of patients \\
\hline Monotherapy & $\mathbf{1 2 8}(\mathbf{7 1 . 1 1 \% )}$ \\
SV & $44(24.4 \%)$ \\
PHT & $33(18.3 \%)$ \\
CBZ & $27(15 \%)$ \\
PBT & $19(10.55 \%)$ \\
DZP & $5(2.78 \%)$ \\
\hline Combination therapy & $\mathbf{6 2 ( 3 4 . 4 4 \% )}$ \\
Dual therapy & \\
CBZ+SV & $15(8.3 \%)$ \\
PHT+PBT & $12(6.67 \%)$ \\
PHT+CZ & $11(6.1 \%)$ \\
SV+LTC & $7(3.88 \%)$ \\
SV+LAM & $4(2.2 \%)$ \\
Three drug therapy & \\
SV+CBZ+OXC & $2(1.1 \%)$ \\
LTC+LAM+GP & $1(0.5 \%)$ \\
\hline SVE Sodum valpon
\end{tabular}

$\mathrm{SV}=$ Sodium valproate, $\mathrm{PHT}=$ Phenytoin, $\mathrm{CBZ}=$ Carbamazepine, $\mathrm{PBT}=$ Phenobarbitone, $\mathrm{DZP}=$ Diazepam, $\mathrm{LTC}=$ Levetiracetam, $\mathrm{LAM}=$ Lamotrigine, $\mathrm{OXC}=$ Oxcarbazepine, $\mathrm{GP}=$ Gabapentin.

In the table 2, the AED regimen of the study subjects has been presented. Majority of patients were under monotherapy (71.11\%) and $34.44 \%$ patients were under combination therapy. Sodium valproate was the most prescribed drug in monotherapy (24.4\%) followed closely by Phenytoin (18.3\%). Most prescribed two drug combination was of Sodium valproate and carbamazepine, in 15 subjects $(8.3 \%)$, followed by Phenytoin and 
Phenobarbitone combination used in 12 subjects(6.67\%). The three drug combination was given for only 3 patients. The preference of monotherapy over polytherapy and conventional AED over novel drugs has also been reported in a research by Soha et al., 2011.

The table 3, the ADRs found in the study has been presented. A total of 56 ADRs were noted in which the CNS adverse effects, $24(42.85 \%)$ ADR cases, were highest in number. Carbamazepine produced memory loss (in 5 cases), aggression and reduced learning power (in 3 cases each). Phenytoin, Phenobarbitone and Sodium valproate produced CNS adverse effects. CNS ADRs like aggression, restlessness, behavioral changes, cognitive impairment by AEDs has been identified and reported in earlier studies (Lieven, 2006; Shobana et al., 2010; Clare et al., 2011; Juny et al., 2013; Jim et al., 2013).

Sodium valproate and Phenytoin produced 3 cases with hepatotoxicity. Combination of Carbamazepine with Sodium valproate developed 3 cases of hepatotoxicity while Phenytoin with carbamezepine combination developed 2 cases of hepatotoxicity.

Hepatotoxic effects of AEDs is well established. Phenytoin and Carbamazepine predominantly undergoes hepatic metabolism by enzyme induction. Production of toxic metabolites, pharmacokinetic changes, therapy with longer duration and underlying liver disease are considered as major causes of AED induced hepatotoxicity. Various studies has revealed the link between AEDs and hepatotoxicity (Gopaul et al.,2003; Kostrubsky et al.,2005; Björnsson , 2008; Hussein et al.,2013). A review study by Ahmed and Siddiqi, 2006, clearly states the pivotal role of AEDs at inducing hepatotoxicity. Hyponatremia was reported in 3 patients who took Carbamazepine and 2 patients who were under sodium valproate therapy respectively. A study by Van et al., 1994, has described that Carbamazepine has led to hyponatremia in patients with epilepsy. Many case reports have identified the role of Sodium valproate and Carbamazepine in developing hyponatremia (Gandelman., 1994; Branten et al., 1998; Miyaoka et al., 2001). Sodium valproate monotherapy induced curling of hair in two cases. Sodium valproate when taken for longer period (more than 4 years) changes the texture of hair. A study conducted by Hoseinali et al., 2005, concluded that younger patients have higher incidence of hair loss and curling and the original and normal texture of hair will appear after discontinuance of sodium valproate. Monotherapy with Phenytoin and Sodium valproate produced one case of skin rash each. This coincides with the study conducted by Ghaffarpour et al., 2005, and they concluded that skin rashes by anti epileptics is more likely to occur during the first few months of treatment. Combination of Sodium valproate with Lamotrigine also developed a case of serious skin rash. A study by Dirk et al., 2007, mentions that children are at increased risk for developing Lamotrigine related skin rash when compared to adults. Sodium valproate interferes with the metabolism of LAM by inhibiting hepatic glucuronides, leading to increased LAM blood levels. According to other studies incidence of Lamotrigine induced rash in epileptic patients is $8-20 \%$ and the chance of developing skin rashes with combination of Sodium Valproate and Lamotrigine has also been proved (Li et al., 1996; Ghaffarpour et al., 2005; Mohammed, 2007). Monotherapy with Sodium valproate caused weight gain in 4 patients. Valproic acid may alter energy intake, physical activity /energy expenditure or both. Alterations in energy intake or expenditure can result from, or be associated with, changes in biological mechanisms, including hormone levels (Martin et al., 2009).

Table 3: ADRs distribution in the subjects $(\mathrm{n}=180)$

\begin{tabular}{|c|c|c|c|c|}
\hline S.No. & Organ system & Type of reaction & AED Therapy & No. of patients with ADR \\
\hline \multirow{11}{*}{1.} & \multirow{11}{*}{ CNS (24) } & \multirow{3}{*}{ Aggression } & Carbamazepine & 3 \\
\hline & & & Sodium valproate & 1 \\
\hline & & & Phenobarbitone & 1 \\
\hline & & \multirow{6}{*}{ Memory loss } & Sodium valproate & 3 \\
\hline & & & Carbamazepine & 5 \\
\hline & & & Phenytoin & 2 \\
\hline & & & Phenobarbitone & 1 \\
\hline & & & $\mathrm{PHT}+\mathrm{PBT}$ & 2 \\
\hline & & & $\mathrm{PHT}+\mathrm{CBZ}$ & 1 \\
\hline & & \multirow{2}{*}{ Reduced learning } & Carbamazepine & 3 \\
\hline & & & Phenobarbitone & 2 \\
\hline \multirow{4}{*}{2.} & \multirow{4}{*}{ Hepatic (11) } & \multirow{4}{*}{ Hepatotoxicity } & Sodium valproate & 3 \\
\hline & & & Phenytoin & 3 \\
\hline & & & $\mathrm{CBZ}+\mathrm{SV}$ & 3 \\
\hline & & & $\mathrm{PHT}+\mathrm{CBZ}$ & 2 \\
\hline \multirow{2}{*}{3.} & \multirow{2}{*}{ Renal (5) } & \multirow{2}{*}{ Hyponatremia } & Carbamazepine & 3 \\
\hline & & & Sodium valproate & 2 \\
\hline \multirow{4}{*}{4.} & \multirow{4}{*}{ Dermatologic (5) } & \multirow{3}{*}{ Skin rashes } & Sodium valproate & 1 \\
\hline & & & Phenytoin & 1 \\
\hline & & & SV+LAM & 1 \\
\hline & & Curling of hair & Sodium valproate & 2 \\
\hline 5. & Endocrine (4) & Weight gain & Sodium valproate & 4 \\
\hline 6. & Muscoskeletal (4) & Hypocalcaemia & Phenytoin & 4 \\
\hline 7. & Blood (3) & Anemia & Phenytoin & 3 \\
\hline
\end{tabular}

PHT=Phenytoin, $\mathrm{PBT}=$ Phenobarbitone, $\mathrm{CBZ}=$ Carbamazepine, $\mathrm{SV}=$ Sodium valproate, $\mathrm{LAM}$ =Lamotrigine. 
Table 4: Demographic, clinical and AED treatment characteristics of patients with and without ADR.

\begin{tabular}{lcc}
\hline Characteristics & Patients with ADRs (n=56) & Patients without ADRs (n=124) \\
\hline Gender & & P value \\
Male, $\mathrm{n}(\%)$ & $37(66.07 \%)$ & $71(57.26 \%)$ \\
Female, $\mathrm{n}(\%)$ & $19(33.93 \%)$ & $53(42.74 \%)$ \\
\hline Age in years (mean \pm SD) $\mathbf{( 2 8 . 7 5} \pm \mathbf{2 1 . 7 6 )}$ & $\mathbf{3 6 . 2 \pm 2 2 . 4 2 )}$ & $\mathbf{( 2 5 . 4 \pm 1 8 . 7 2 )}$ \\
$0-14$ & $13(23.3 \%)$ & $46(37.1 \%)$ \\
$15-29$ & $12(21.4 \%)$ & $34(27.4 \%)$ \\
$30-44$ & $8(14.3 \%)$ & $23(18.5 \%)$ \\
$45-59$ & $11(19.6 \%)$ & $12(9.7 \%)$ \\
$60-74$ & $12(21.4 \%)$ & $9(7.3 \%)$ \\
Types of Seizure & & \\
Generalised tonic clonic & $36(64.30 \%)$ & $48(38.70 \%)$ \\
Myoclonic & $7(12.50 \%)$ & $21(16.90 \%)$ \\
Atonic & $0(0.00 \%)$ & $0(0.00 \%)$ \\
Status epilepticus & $0(0.00 \%)$ & $0(0.00 \%)$ \\
Complex partial & $8(14.30 \%)$ & $23(18.60 \%)$ \\
Unclassified & $5(8.90 \%)$ & $23(18.60 \%)$ \\
\hline AED Treatment Regimen & & \\
Monotherapy & $47(83.93 \%)$ & $81(65.32 \%)$ \\
Combination therapy & $9(6.07 \%)$ & $43(34.68 \%)$ \\
\hline
\end{tabular}

Similar findings were observed in other studies also (Biton, et al., 2001; El-Khatib, et al., 2007; Kanemura et al., 2012). Monotherapy with Phenytoin caused hypocalcaemia in 4 and anemia in 3 patients respectively. Phenytoin induced folic acid deficiency has been studied earlier by Ryan and Forshaw, 1955; and a review by Abhishek et al, 2012, backs our result. Several studies shown that the serum concentration of the active vitamin D metabolites is diminished after administration of Phenytoin and other anticonvulsant drugs. This effect of these drugs is due to induction of hepatic metabolic enzymes thus resulting in an acceleration of vitamin D metabolism. Thus these drugs have an indirect effect on calcium homeostasis (Taghi et al., 1991; Melinda et al., 2007).

In the table 4, demographic, clinical and AED treatment characteristics of patients with and without ADR was presented. In this study male subjects were dominant, with ADRs 37(66.07\%) and without ADRs 71(57.26\%) but does not show any statistical significance $(\mathrm{p}=0.3246)$. This result was in contrast to that of Roopa et al., 2008 and Soha et al., 2011. Increase in age will contribute to the chance in developing ADR. This may be due to alteration in pharmacokinetics and pharmacodynamics of AED. The mean \pm SD age of the patients with ADR was higher than that of patients without ADR $(36.2 \pm 22.42$ vs $25.4 \pm 18.72$ years respectively) and this difference was shown statistical significance also in our study $(\mathrm{p}<0.0001)$. This coincides with the study of Roopa et al., 2008, where the age distribution showed statistically significant difference in occurrence of ADRs. Link between the type of seizure with ADRs did not show significance in this research $(p=0.1796)$. Also the significance in the relation between the type of AED regimen (monotherapy and combination therapy) and reported number of ADRs was not achieved among the subjects $(\mathrm{p}=0.0126)$. This coincides with the study of Soha et al., 2011. The causality assessment of ADRs by using Naranjo's was done. ADR assessment scale revealed that 19 cases of ADR had definite relation with the drug while 22 cases had probable relation and 13 cases had possible relation with the drug. Numerous side effects were also observed among the patients. 170 patients were having sedation with Phenobarbitone, Carbamazepine, Sodium Valproate, Phenytoin Oxcarbamazepine, Lamotrigine, and Diazepam. 93 patients suffered gastric irritation with Sodium Valproate, Phenytoin, Carbamazepine. Other known side effects like drowsiness, dizziness, nausea, headache and hair loss were also observed among the study subjects. All the observed side effects were brought to attention through earlier studies and are expected while using AEDs (Robert, 2000; Walia et al., 2004; Roop et al., 2008; Soha et al., 2011; Jim et al., 2013; Mansur et al., 2013).

\section{CONCLUSION}

Despite the novel discoveries and recent advances in the treatment and control of epilepsy, traditional drugs like Sodium valproate, Phenytoin, and Carbamazepine still remain as the most sought choice in the management of seizures in epileptic patients in India. In our study on AED induced adverse drug reactions, we observed that there was a predominance of male epileptic patients. Majority of the enrolled patients were adults. Majority of subjects were affected with generalized type of seizure compared to partial seizure. It was observed that monotherapy with Sodium valproate was higher and it was the most preferred drug in most cases, closely followed by Carbamazepine and Phenytoin, and the differences in the preferences of these drugs were, in fact, very negligent. It may further be added that conventional anti epileptic drugs can be accounted for better seizure control and cost efficacy. Moreover, Sodium valproate was found to induce more number of ADRs followed by Carbamazepine and Phenytoin. CNS related ADRs were detected in most of the cases followed by hepatotoxicity. Treatment with antiepileptic drugs was continued in all cases, except in three cases of ski rashes, with Phenytoin and Sodium valproate monotherapy, respectively. The third with Lamotrigine + Sodium valproate combination therapy.

In India, the skills of pharmacists, in clinical side of health care sector, are underutilized and this can be a reason for under reporting of ADRs. With proper training and exposure, 
Pharmacists can contribute to better adherence of treatment regimen/schedule and can contribute to minimization of ADRs. It should be emphasized that this was a small size study. Therefore our results must further be substantiated and confirmed with a larger size of study subjects.

\section{ACKNOWLEDGEMENT}

We acknowledge the management of J.K.K.Nattraja College of Pharmacy, Erode, for their immense support. We express our gratitude to Dr. Hrishi Varayathu for his valuable time, expertise knowledge and suggestions which played as a crucial support system to finish this study.

\section{REFERENCES}

Abbashar Hussein, Amira Abdulgalil, Faroug Omer, Hassan Eltoum, Ahmed Hamad, Omer El-Adil, Bedraldin Mubarak, Mohmad Malkaldar, Iway Idris, Yasin Alwidaa, Esam Mahmoud. Correlation between Serum Level of Antiepileptic Drugs and their Side Effects. Oman Med J, 2010; 25(1): 17-21.

Abhishek SN, B. Nataraju and G. T. Subhas. Phenytoin-Folate Interactions: How Far is Safe Folate Supplementation in Phenytoin Treated Epileptic Patients? J.App.Pharm.Sci, 2012; 02(06): 230-235.

Ahmed SN, Siddiqi ZA. Antiepileptic drugs and liver disease. Seizure, 2006; 15(3): 156-164.

Amrita S, Kamalesh D, Nilay KD, Dibyendu C, Goutameswar M, Santanu KT. Use of anti-epileptic drugs in a tertiary care hospital of Eastern India with emphasis on epilepsy due to neurocysticercosis. Indian J Pharmacol, 2012; 44(1): 106-110.

Biton V, Mirza W, Montouris G, Vuong A, Hammer AE, Barrett PS. Weight change associated with valproate and lamotrigine monotherapy in patients with epilepsy. Neurology, 2001; 56(2): 172-177.

Björnsson E. Hepatotoxicity associated with antiepileptic drugs. Acta Neurol Scand, 2008; 118(5): 281-290.

Branten AJ, Wetzels JF, Weber AM, Koene RA. Hyponatremia due to sodium valproate. Ann Neurol, 1998; 43(2): 265-267.

Clare M. Eddy, Hugh E. Rickards and Andrea E. Cavanna. The cognitive impact of antiepileptic drugs. Ther Adv Neurol Disord, 2011; 4(6): 385-407.

Dirk D, Hassan AH, Boulenouar M, Hisham AM. Short-term efficacy and safety of valproate sustained release formulation in newly diagnosed partial epilepsy. Saudi Med J, 2007; 28(9): 1402-1407.

El-Khatib F, Rauchenzauner M, Lechleitner M, et al. Valproate, weight gain and carbohydrate craving: a gender study. Seizure, 2007; 16(3): 226-232.

Gandelman MS. Review of carbamazepine-induced hyponatremia. Prog Neuropsychopharmacol Biol Psychiatry, 1994; 18(2): 211-233.

Ghaffarpour M, Hejazie SS, Harirchian MH, Pourmahmoodian H. Phenytion, carbamazepine, sodium valproate and lamotrigine induced cutaneous reactions. Acta Med Iran, 2005; 43(1): 37-42.

Hoseinali E, Sadollah S and Shahriar SE. Frequency of Sodium Valproate - Induced Hair Loss and Curly Hair. Iranian J Pharmacol Thera, 2005; (2): 143-145.

HS Lee, SY Wang, Donald MS, CC Wang, SJ Chen, HC Fan. The impact of the use of antiepileptic drugs on the growth of children. BMC Pediatr, 2013; 13:211.

Jim T, Stacy EM, Brooks BP. Behavioral Side Effects of Antiepileptic Drugs. US Pharm, 2013; 38(11): 15-20.

Juny S, Adepu R, Keshava BS, Harsha S. Assessment of antiepileptic drugs usage in a South Indian tertiary care teaching hospital. Neurol Asia, 2013; 18(2): 159-165.

Kanemura H, Sano F, Maeda Y, Sugita K, Aihara M. Valproate sodium enhances body weight gain in patients with childhood epilepsy: a pathogenic mechanisms and open-label clinical trial of behavior therapy. Seizure, 2012; 21(7): 496-500.

Kostrubsky SE, Sinclair JF, Strom SC, Wood S, Urda E, Stolz DB, Wen YH, Kulkarni S, Mutlib A. Phenobarbital and phenytoin increased acetaminophen hepatotoxicity due to inhibition of UDPglucuronosyltransferases in cultured human hepatocytes. Toxicol Sci, 2005; 87(1): 146-155.

Li LM, Russo M, Donoghue MF, Sander J. Allergic skin rash with lamotrigine and concomitant valproate therapy. Arq Neuropsiquiatr, 1996; 54(1): 47-49.

Lieven Lagae. Cognitive side effects of anti-epileptic drugs. The relevance in childhood epilepsy. Seizure, 2006; 15: 235-241.

Mansur H, Sharifuddin K, Azhahul H, et al. Antiepileptic drug utilization in Bangladesh: experience from Dhaka Medical College Hospital. BMC Res Notes, 2013; 6: 473-476.

Martin CK, H Han, SD Anton, FL Greenway, and SR Smith. Effect of valproic acid on body weight, food intake, physical activity and hormones: results of a randomized controlled trial. J Psychopharmacol, 2009; 23(7): 814-825.

Melinda von BS, Kristi C, Rosita RP, Theresa MF. Effects of phenytoin and carbamazepine on calcium transport in Caco-2 cells. Toxicol in Vitro, 2007; 21(5): 855-862.

Miyaoka T, Seno H, Itoga M, Kishi T, Ishino H, Horiguchi J. Contribution of sodium valproate to the syndrome of inappropriate secretion of antidiuretic hormone. Int Clin Psychopharmacol, 2001; 16(1): 59-61.

Mohammed MJ. Potentially serious Lamotrigine-related skin rash. Neuroscience, 2007; 12(1): 17-20.

RRS Hussein, Rasha HS, Ahmed MAA, Mona HT, Mohamed EAA. Effect of antiepileptic drugs on liver enzymes. Beni-Suef Univ J App Sci, 2013; 2(1): 14-19.

Robert SG. Adverse Effects of Antiepileptic Drugs. Epilepsia, 2000; 41(2): 42-52.

Roopa B.S, Narayan SS, Sharma GR, Rodrigues RJ, Kulkarni C. Pattern of adverse drug reactions to anti-epileptic drugs: a crosssectional one-year survey at a tertiary care hospital. Pharmacoepidemiol Drug Saf, 2008;17(8): 807-812.

Ryan GMS and JWB Forshaw. Megaloblastic Anaemia Due to Phenytoin Sodium. Br Med J, 1955; 2(4933): 242-243.

S Gopaul, K Farrell, F Abbott. Effects of age and polytherapy, risk factors of valproic acid (VPA) hepatotoxicity, on the excretion of thiol conjugates of (E)2,4 diene VPA in people with epilepsy taking VPA. Epilepsia, 2003; 44 (3): 322-328.

Shobhana M, Sumana S, Ramesh L, Satish KM. Utilization pattern of antiepileptic drugs and their adverse effects, in a teaching hospital. Asian J Pharm Clin Res, 2010; 3(1): 55-59.

Soha N, Afshin BH, Iman K. Adverse Reactions to Antiepileptic Drugs in Epileptic Outpatients: A Cross-Sectional Study in Iran. Clin Neuropharm, 2011; 4(2): 79-83.

Swetha M, Mohammad A, Vasant. RC, Raghunandan M. Antiepileptic Drugs Utilization Pattern \& Their Adverse Drug Reactions in a Teaching Hospital in South India. Asian J Biochem Pharmaceut Res, 2013; 3(1): 205-214.

Taghi G, Hamid R J, Abbas G. Anticonvulsant therapy-induced Alterations in calcium homeostasis. Med J Islam Repub Iran, 1991; 5(3): 135-137.

Van AT, Bakshi R, Devaux CB, Schwabe S. Hyponatremia associated with carbamazepine and oxcarbazepine therapy: a review. Epilepsia, 1994; 35(1): 181-188.

Walia KS, Khan EA, Ko DH, Raza SS, Khan YN. Side effects of antiepileptics--a review. Pain Pract, 2004; 4(3): 194-203.

\section{How to cite this article:}

Keerthy J, Kavitha P, Sambathkumar R, Sruthi A. A Study on the Adverse Drug Reactions Induced by Anti Epileptic Drugs in the Epileptic Patients. J App Pharm Sci, 2016; 6 (05): 119-123. 